\title{
An Unusual cause for Vocal Cord Palsy: Case Record
}

\section{Bipin Thomas Varghese, Paul Sebastian, GM Divya}

\section{ABSTRACT}

Objectives: We reporta case of granulomatous lesion infiltrating the recurrent laryngeal nerve to produce vocal cord palsy, the diagnostic dilemma posed by it and its management.

Materials and methods: Clinical documentation of a 52-yearold man who presented with hoarseness of 3 months duration is presented. The clinical examination revealed immobile left vocal cord and a bulky (Lt) ventricular band with a suspicious ulcer on its posterior aspect. A computed tomographic (CT) scan of the neck and chest revealed a $1 \times 1 \mathrm{~cm}$ nodular lesion in the tracheoesophageal groove.

Conclusion: Causes forvocal cord palsies are wide ranging and predominantly include malignant infiltration of the vagus or the recurrent laryngeal nerve. The anatomical site of involvement can be clinically and radiologically assessed with ease. Vocal cord palsy due to recurrent laryngeal nerve involvement by a granulomatous lesion is an unreported phenomenon and we believe that this is the first case to be reported in literature.

Summary: An unusual case of recurrent laryngeal nerve granuloma is reported with the diagnostic dilemma that it presented. This was successfully managed by surgical excision and postoperative antituberculosis treatment.

Keywords: Vocal cord palsy, Recurrent laryngeal nerve, Granuloma, Extrapulmonary tuberculosis.

How to cite this article: Varghese BT, Sebastian P, Divya GM. An Unusual cause for Vocal Cord Palsy: Case Record. Int J Phonosurg Laryngol 2012;2(2):88-90.

\section{Source of support: Nil}

Conflict of interest: None declared

\section{INTRODUCTION}

Commonest cause for unilateral vocal cord fixity is infiltration of the recurrent laryngeal nerve ( $R L N)$ by malignancy. Clinical examination and imaging localizes the site of infiltration and fine needle aspiration cytology (FNAC) often yields the diagnosis. O ccasionally when the FNAC is inconclusive or when it is difficult to differentiate between a poorly differentiated carcinoma and lymphoma an open biopsy may be required.

We present a case of left vocal cord palsy in an adult male which was due to a circumscribed lesion in the tracheoesophageal groove, the FNAC of which was repeatedly inconclusive. With a high index of suspicion of neurogenic tumor of the RLN , excision of the lesion was planned and to our surprise it was reported by the pathologist as a granulomatous lesion and clinically confirmed to be extrapulmonary tuberculosis.

\section{CASE REPORT}

A 52-year-old man presented with hoarseness of 3 months duration to our outpatient department on 22nd November 2002. He denied history of swallowing difficulty, breathlessness, earache, episodes of choking spells or cough or any previous surgical interventions in the neck or trauma to the head and neck. On examination he was well-built and nourished and all there were no positive systemic findings. The clinical examination of neck was grossly normal except for a $2 \times 1 \mathrm{~cm}$ flat superficial level two node. Indirect laryngoscopy (IL) showed an immobile left vocal cord and a bulky ( $\mathrm{Lt}$ ) ventricular band with a suspicious ulcer on its posterior aspect and the postnasal examination was normal. A computed tomographic (CT) scan of the neck and chest revealed a $1 \times 1 \mathrm{~cm}$ nodular lesion in the tracheoesophageal groove (Fig. 1). U Itrasound-guided FNAC of the lesion was inconclusive and plain FNAC of the level two node showed reactive hyperplasia. A direct laryngoscopic (DL) examination under local anesthesia confirmed the IL findings and biopsy was taken from the suspicious area which turned out to be inconclusive.

W ith a provisional diagnosis of a neurogenic tumor from the RLN the patient was taken up for direct laryngoscopy and hypopharyngoscopy and surgical excision of the nodular lesions under general anesthesia on 19th may 2003 after explaining the uncertainty of further course of treatment and the possibility of recovery of the vocal cord palsy. A suspicious area in the $(\mathrm{Lt})$ retroarytenoid area was biopsied during the rigid endoscopy which was otherwise normal and on neck exploration via a hockey stick incision he was found to have a nodular firm mass that was apparently inseparable from the RLN (Figs $2 A$ to $C$ ). The mass which clearly looked like a nerve sheeth tumor was carefully separated from the RLN leaving the nerve in continuity. The level two node was also excised (Fig. 3). The histopathology reports were that of a granulomatous lesion and granulomatous Iymphadenitis, contrary to our expectation.

A M antoux test carried out was positive. Hewas referred to the chest physician who commenced antituberculosis treatment for extrapulmonary tuberculosis. For the vocal cord palsy he was adviced vocal enhancement exercises. He was disease free during the follow-up for 1 year.

The case is presented to highlight an absolutely rare cause for vocal cord palsy and diagnostic and therapeutic dilemma it presented. High index of suspicion, judicious 


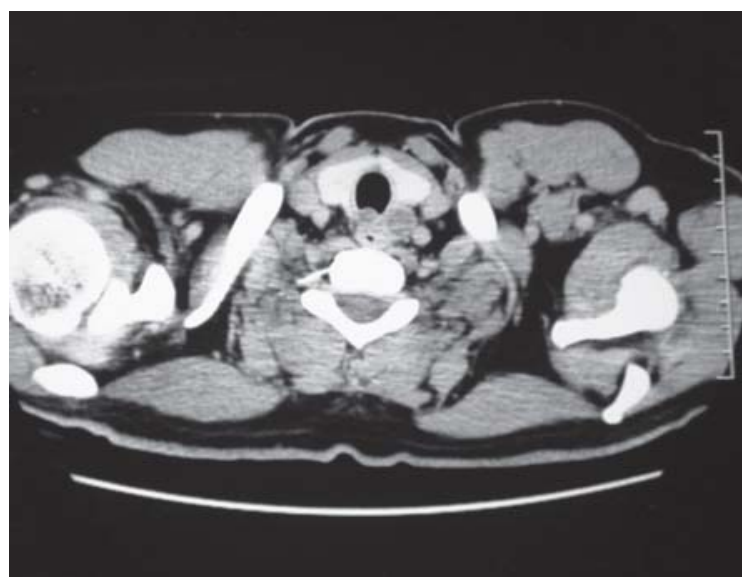

Fig. 1: CT scan of the root of neck and chest showing the lesion

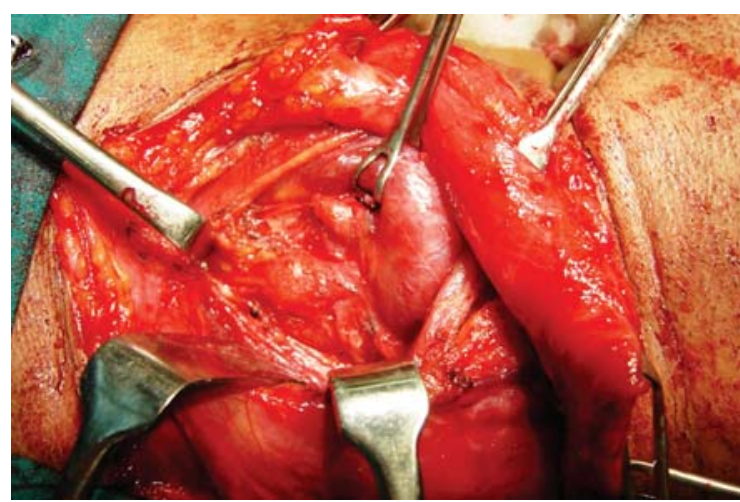

Fig. 2A: Preoperative view of nodular mass

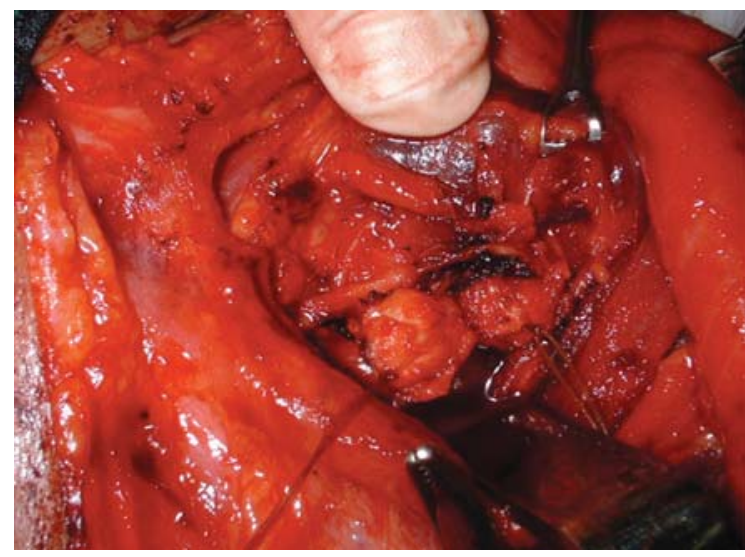

Fig. 2B: Nodular mass separated from RLN with difficulty

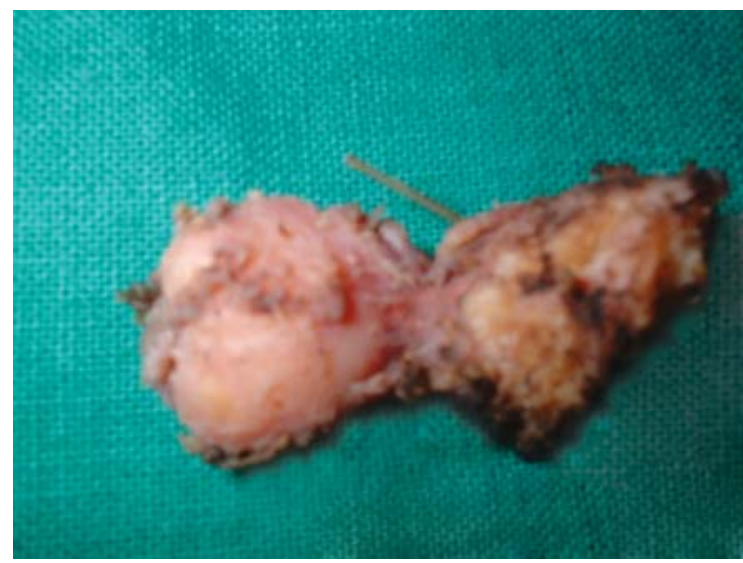

Fig. 2C: Lesion after excision

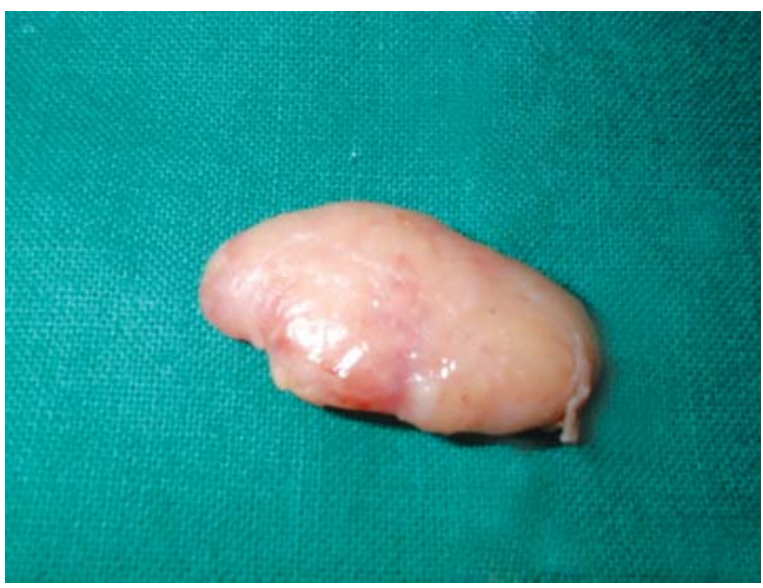

Fig. 3: Excised level 2 node

surgical intervention and clinicopathological correlation helped us in the optimal and successful management. An extensive review of literature did not reveal any other similar case and we believe this to be the first case report of a tuberculous RL N granuloma.

\section{DISCUSSION}

The commonest cause for unilateral fixity of vocal cord is its paralysis due to temporary or permanent damage to the $\mathrm{RLN}$ or vagus nerve due to malignant infiltration, trauma, idiopathic causes, neurological abnormalilty, infections or inflammations (Table 1 ). In a recent update of review of causes of vocal cord palsy by Watkinson et $\mathrm{al}^{1} 25 \%$ of all cases were due to malignancy, one-half being caused by lung cancer, $20 \%$ by cancer of the esophagus, $10 \%$ by thyroid cancers and $20 \%$ by gliomas, nasopharyngeal cancers and lymphomas. Surgical trauma which constitutes

Table 1: Causes of vocal cord fixity

Malignant: Bronchogenic carcinoma, esophageal cancer, thyroid and parathyroid malignancies, metastatic neck and mediastinal nodes.

Surgical/Traumatic: Thyroidectomy parathyroidectomy, partial laryngectomy, radical neck dissection, removal of pharyngeal pouch, pneumonectomy, coronary artery bypass grafting (CABG), carotid endarterectomy, penetrating neck or chest trauma, postintubation, whiplash injuries, posterior fossa surgery and spine fusion by anterior approach.

\section{Idiopathic}

Inflammatory/Infectious: Syphilis, pulmonary tuberculosis, thyroiditis, viral (influenza and infectious mononucleosis).

Neurological: Wallenberg syndrome, syringomyelia, encephalitis, Parkinsons disease, poliomyelitis, multiple sclerosis (MS), myasthenia gravis, amyotrophic lateral sclerosis (ALS), progressive bulbar palsy, diabetic/alcoholic neuropathy and Guillain-Barre syndrome.

Miscellaneous: Neurogenic tumors of RLN or vagus, rheumatoid arthritis, hemolytic anemia, syphilis and collagen diseases, sarcoidosis, lupus, polyarteritis nodosa, Ortner's syndrome (left atrial hypertrophy), aortic aneurysm, thrombosis of subclavian vein and granulomas. 
$20 \%$ of all cases closely follows the list which again is followed by nonsurgical trauma to the nerve (15\%), neurological causes (15\%), and idiopathic causes (15\%). Only $5 \%$ of cases are due to infective and inflammatory causes and miscellaneous causes form another $5 \%$.

Although the approach to the management of vocal cord palsy is well known, ${ }^{1}$ quite unusually we may be faced with a diagnostic and therapeutic dilemma where individualized decisions may be needed to manage the case. The present case which mimicked malignancy is an example. The differential diagnosis (DD) of a well-circumscribed tumor in the tracheoesophageal groove ${ }^{2}$ are metastatic node(s), tuberculous adenitis, ${ }^{3}$ parathyroid adenoma and cyst, ${ }^{4}$ neurofibroma of the RLN. ${ }^{5}$ Although the 1st three possibilities were clinically and radiologically excluded to arrive at a working diagnosis of neurogenic tumor of the $R L N$, the HPR of granulomatous lesion was a surprise.

An extensive M edline search of the literature did not yield any reports of granulomatous lesion of the recurrent laryngeal nerve, although tuberculomas of other peripheral nerves werefound. ${ }^{6,7}$ Commonest cause for peripheral nerve granuloma in our part of the world is tuberculosis and this needs to be excluded before other causes are considered. Thorotrast granuloma ${ }^{8}$ teflonoma ${ }^{9}$ and sarcoidosis ${ }^{10}$ are the other rare possi bilities to be considered in the case presented.

\section{REFERENCES}

1. Watkinson J C, Gaze M N, W ilson JA. Rehabilitation of speech and swallowing. In: Stell and M arans (Eds). Head and neck surgery (4th ed). Hodder A rnold 2008;2:362.

2. $M$ afee $M F, V$ alvassori $G E, B$ ecker $M$. V alvassoris imaging of the head and neck (2nd ed). Illustrated published by Thieme 2004.

3. Nakamura M, Fujishima S, Hori S, Nakamura H, Namiki M, Takahashi $M$, et al. A $n$ adult case of cervico-mediastinal tuberculous lymphadenitis. N ihon K okyuki Gakkai Zasshi 2000 M ar;38(3):223-28.
4. Sen P, Flower N, Papesch M, Davis A, Spedding A V . A benign parathyroid cyst presenting with hoarse voice. J Laryngol O tol 2000;114(2):147-48.

5. Contant A, A cquaviva F, Peral di R, Fanton Y. N eurofibroma of the recurrent nerve: A propos of a case. R ev L aryngol Otol R hinol (B ord). 1995; 116(3):225-28.

6. Nucci $F, M$ astronardi $L$, A rtico $M$, Ferrante $L$, A cqui $M$. Tuberculoma of the ul nar nerve: C ase report. N eurosurgery 1988 M ay;22(5):906-07.

7. Orrell RW, King RH, B owler JV, Ginsberg L. Peripheral nerve granuloma in a patient with tuberculosis. J Neurol Neurosurg Psychiatry 2002 Dec;73(6):769-71.

8. K uilman J. Thorotrast granuloma of the pharynx with left total recurrent paralysis, 18 years after angiography. Pract Otorhinolaryngol (B asel) 1962;24:121-24.

9. Wassef M, A chouche J, Guichard J P, Tran B a H uy P. A delayed teflonoma of the neck simulating a thyroid neoplasm. ORL J Otorhinolaryngol Relat Spec $1994 \mathrm{~N} \mathrm{ov}-\mathrm{Dec} ; 56(6): 352-56$.

10. Ogawa $Y$, Higuchi E, K oga H, Tanaka Y, Tokisawa S, Rikimaru $\mathrm{T}$, et al. A case of sarcoidosis presenting with hoarseness and dysphagia due to glossopharyngeal and vagus nerve paresis. Nihon K yobu Shikkan Gakkai Zasshi 1994 J un;32(6):602-05.

\section{ABOUT THE AUTHORS}

\section{Bipin Thomas Varghese (Corresponding Author)}

A dditional Professor, Department of Surgical Oncology (Head and Neck Services), Regional Cancer Centre, Thiruvananthapuram Kerala, India, Phone: 91-471-2442541, Fax: 91-471-2445474 e-mail: bipintv@gmail.com

\section{Paul Sebastian}

Professor, Department of Surgical Oncology (Head and Neck Services), Regional C ancer Centre, Thiruvananthapuram, K erala, India

\section{GM Divya}

Senior Resident, D epartment of Surgical Oncology (Head and Neck Services), Regional C ancer Centre, Thiruvananthapuram, K erala, India 